Cahiers $d u$ MONDE RUSSE

\section{Cahiers du monde russe}

Russie - Empire russe - Union soviétique et États indépendants

$47 / 4 \mid 2006$

Varia

\title{
Oleg Kutafin, Rossijskaja avtonomija
}

\section{Françoise Daucé}

\section{OpenEdition \\ Journals}

Édition électronique

URL : https://journals.openedition.org/monderusse/6791

DOI : 10.4000/monderusse. 6791

ISSN : $1777-5388$

Éditeur

Éditions de l'EHESS

Édition imprimée

Date de publication : 30 décembre 2006

Pagination : 922-925

ISBN : 978-2-7132-2098-2

ISSN : $1252-6576$

\section{Référence électronique}

Françoise Daucé, «Oleg Kutafin, Rossijskaja avtonomija », Cahiers du monde russe [En ligne], 47/4 2006, mis en ligne le 03 juillet 2009, consulté le 02 septembre 2022. URL : http://

journals.openedition.org/monderusse/6791 ; DOI : https://doi.org/10.4000/monderusse.6791

Ce document a été généré automatiquement le 2 septembre 2022.

Tous droits réservés 


\title{
Oleg Kutafin, Rossijskaja avtonomija
}

\author{
Françoise Daucé
}

\section{RÉFÉRENCE}

Oleg KUTAFIN, Rossijskaja avtonomija [L'autonomie russe]. Moscou : Prospekt, 2006, $768 \mathrm{p}$.

1 L'ouvrage d'oleg Kutafin est le fruit d'un travail encyclopédique exhaustif sur la notion juridique d'« autonomie » en Russie, de l'Empire à la Fédération postsoviétique. Le terme même d'« autonomie ", fréquemment utilisé à la période soviétique (républiques et oblasti autonomes), doit être compris en français comme « la possibilité de décider accordée à un organisme par rapport à un pouvoir central ». Ce livre d'un volume impressionnant (plus de 700 pages) s'efforce de montrer les différentes acceptions que ce terme recouvre dans le droit public russe et de présenter tant l'histoire du concept d'autonomie en Russie que les modalités juridiques de sa mise en application dans le pays. Selon l'auteur, «l'idée d'autonomie a été comprise différemment tout au long du xIX ${ }^{e}$ puis du $\mathrm{Xx}^{\mathrm{e}}$ siècle ». Par son statut juridique, on peut cependant dire qu'elle occupe une place entre l'État unitaire, au sein duquel elle se trouve, et l'« 'auto-administration' locale». CEuvre d'un juriste académicien, l'ouvrage fait preuve d'une grande rigueur dans la citation des auteurs, des dates, des textes de lois. Plus descriptif qu'analytique, il pourrait devenir un ouvrage de référence sur la question des nationalités et des territoires autonomes en Russie. L'absence de commentaires personnels ou de remarques critiques de l'auteur invite néanmoins le lecteur à mener seul une réflexion historique et juridique à partir des données fournies.

2 Suivant un plan chronologique strict, $\mathrm{O}$. Kutafin rappelle que c'est à la fin du XIX ${ }^{e}$ siècle que les débats juridiques autour de la notion d'autonomie se développent en Russie. Dans l'esprit des juristes de l'époque, s'inspirant des exemples anglais ou australien, l'autonomie "suppose le droit à une législation autonome ». Au sein de l'Empire russe, aucune forme d'autonomie de ce type n'est juridiquement reconnue. Cependant, les juristes russes admettent qu' « un certain nombre de territoires qui étaient auparavant indépendants (samostojatel'nye) ou qui dépendaient d'une autre puissance ont conservé 
leurs lois locales et bénéficié ainsi d'une autonomie locale (mestnaja avtonomija) plus ou moins large ». C'est le cas de la Pologne, de la Transcaucasie ou de la Finlande. C'est aussi le cas de certains peuples qui ont vu leurs coutumes reconnues et bénéficient d'un statut spécial (inorodcy), comme les Kalmuks de Sibérie, les Kirghizes ou les juifs. Le monarque russe représente cependant un pouvoir d'État unifié et illimité. Son pouvoir législatif l'emporte toujours sur celui des régions qui jouissent d'un degré d'autonomie. Ces dernières ne peuvent apparaître comme une menace pour l'unité de la Russie : il ne s'agit pas d'une fédération entre des partenaires égaux. Comme l'explique en 1905 le juriste F.F.Kokoškin, il ne faut pas confondre "autonomie " et "fédération ». Dans une fédération, les territoires fédérés ont non seulement leur propre législation mais aussi leur propre gouvernement (pravitel'stvo), indépendant du pouvoir central. Le territoire autonome ne peut avoir un tel gouvernement. Le pouvoir exécutif y est représenté par un intendant (namestnik) ou un gouverneur-général, nommé par le chef de l'État.

3 Avant même la révolution de 1917, ces débats juridiques autour de la notion d'autonomie régionale intéressent les leaders bolcheviques. Ils connaissent vraisemblablement les travaux des publicistes de leur temps, qui, dès 1906, insistent sur la nécessité de donner une autonomie aux peuples numériquement importants dotés d'une culture multiséculaire. Dans un ouvrage de 1908, Kokoškin propose de nommer « district autonome " (avtonomnyj kraj) et région autonome (avtonomnaja oblast') les territoires bénéficiant d'un pouvoir législatif propre. Il introduit des termes qui seront repris à la période soviétique. Des permanences semblent donc décelables entre les conceptions juridiques russes et soviétiques, même si l'auteur se contente d'exposer les faits sans chercher à en montrer les continuités. 0 . Kutafin rappelle les débats théoriques après 1917 entre juristes soviétiques sur la notion d'autonomie régionale en présentant en premier, fidèle à la méthodologie soviétique, les textes de Lenin ! Ce dernier défend l'idée d'une autonomie pour les régions se différenciant par les spécificités de leur économie, des traditions ou de la composition nationale. La dimension nationale est placée sur le même plan que l'économie ou les traditions (byt). C'est là une évolution importante. Dans le sillage des chefs bolcheviques, les juristes soviétiques se différencient de leurs prédécesseurs en définissant les territoires autonomes non seulement en fonction de critères nationaux mais aussi de critères économiques et liés à la vie quotidienne. Pour le juriste V.N. Durdenevskij, en 1929, l'autonomie est un moyen pour "éteindre » la question nationale. Le prolétariat exige, pour les plus faibles économiquement et les plus " attardés » culturellement, une " autonomie nationalo-culturelle » qui leur permettra de combler leur retard. Cette autonomie est élaborée dans le cadre strict du centralisme démocratique. Stalin précise les attributions qui doivent demeurer entre les mains du Conseil des commissaires du peuple : affaires militaires, politique extérieure, chemins de fer, poste, monnaie, accords commerciaux, politique économique, financière et bancaire. Écoles, justice, administration pourront dépendre des commissaires du peuple locaux. Stalin n'envisage le fédéralisme en Russie que comme une étape transitoire vers l'unitarisme socialiste.

4 Dans un premier temps, la révolution d'Octobre a surtout conduit au renforcement des revendications nationales et des mouvements de libération des peuples. La République soviétique de Russie devient fédérative et tente d'intégrer en qualité de républiques nationales autonomes les anciens confins de l'Empire. La Constitution de la RSFSR, adoptée en juillet 1918, reconnaît le principe fédératif. À la fin de l'année 1918 apparaît une nouvelle forme d'autonomie : la commune ouvrière (trudovaja kommuna) et, à partir de 1920, la région autonome (avtonomnaja oblast'). En 1918 est créée la commune ouvrière 
des Allemands de la Volga (elle sera supprimée en 1923, comme celle de Carélie, et transformée en république autonome). Des Républiques socialistes soviétiques autonomes (ASSR) sont créées à partir de 1919. De nombreux changements de statuts ont lieu dans les années 1920 et 1930 : transformations de régions autonomes en républiques autonomes, et de républiques autonomes en républiques de l'Union (en Asie centrale). Les juristes soviétiques débattent de ces différentes formes d'autonomie tout au long des années 1920 et 1930, mais aussi après la Seconde Guerre mondiale et jusqu'aux années 1970.

5 La perestroïka gorbatchévienne met fin provisoirement à l'organisation fédérale soviétique et arrache son plus sincère commentaire à Oleg Kutafin: «L'aspiration de toutes les républiques de l'Union à l'indépendance a conduit à une tragédie : l'éclatement de l'Union soviétique ». Cette vision dramatique de la disparition de l'URSS n'est pas sans rappeler les propos du président Putin lors de son adresse à l'assemblée fédérale au printemps 2006. Reprenant ensuite la distanciation juridique qu'il s'impose, l'auteur traite uniquement du devenir des territoires nationaux au sein de la RSFSR. Il rappelle qu'en Russie, certaines républiques autonomes ont manifesté le souhait de devenir souveraines, et certaines régions autonomes celui d'améliorer leur statut. Il convient de souligner que l'auteur ne s'arrête à aucun moment sur le cas particulier de la république de Tchétchénie, engagée dans deux guerres successives contre le pouvoir fédéral. En 1991, les républiques perdent l'adjectif "autonome» et toutes les régions autonomes obtiennent le statut de république à l'exception de la région autonome juive. Les républiques adoptent des déclarations sur leur souveraineté d'État. Le pouvoir fédéral favorise alors cette évolution, y voyant le moyen de prévenir l'éclatement de la Fédération de Russie. La signature de traités bilatéraux entre les sujets de la Fédération et le centre fédéral se situe dans cette perspective. Un tournant intervient cependant en 1999 avec la tentative de reprise en main des régions par le centre fédéral, aboutissant, selon l'auteur, à une perte de souveraineté ramenant les républiques à leur situation de la période soviétique.

6 Le dernier chapitre de l'ouvrage, consacré au concept d'autonomie nationale déterritorialisée, témoigne cependant des innovations intervenues depuis 1991. Partant du constat qu'une nationalité ne peut pas toujours correspondre précisément à un territoire, juristes et hommes politiques russes ont introduit, dans une loi adoptée en 1996, la notion juridique d'autonomie nationale culturelle (NKA, nacional'no-kul'turnaja avtonomija), qui constitue la base d'une définition extraterritoriale des groupes ethniques. La NKA est une forme d'association regroupant des membres d'une communauté et bénéficiant de l'aide publique pour ses activités de promotion culturelle (enseignement, conservation des traditions...). Pour expliquer ce concept, les juristes russes contemporains reconnaissent s'être inspirés des travaux des socialistes autrichiens (R. Springer, O. Bauer) de la fin du XIX ${ }^{e}$ siècle. La notion de NKA avait à l'époque été rejetée par Lenin qui craignait que ce principe ne favorisât le nationalisme. L'auteur clôt son livre sur cette notion nouvelle, laissant au lecteur le soin de la critique ou de la réflexion politique sur les évolutions juridiques présentées. 